\title{
Nanotopographical Cues Augment Mesenchymal Differentiation of Human Embryonic Stem Cells
}

\author{
Emmajayne Kingham, Kate White, Nikolaj Gadegaard, Matthew J. Dalby, \\ and Richard O. C. Oreffo*
}

The production of bone-forming osteogenic cells for research purposes or transplantation therapies remains a significant challenge. Using planar polycarbonate substrates lacking in topographical cues and substrates displaying a nanotopographical pattern, mesenchymal differentiation of human embryonic stem cells is directed in the absence of chemical factors and without induction of differentiation by embryoid body formation. Cells incubated on nanotopographical substrates show enhanced expression of mesenchymal or stromal markers and expression of early osteogenic progenitors at levels above those detected in cells on planar substrates in the same basal media. Evidence of epithelial-to-mesenchymal transition during substrate differentiation and DNA methylation changes akin to chemical induction are also observed. These studies provide a suitable approach to overcome regenerative medical challenges and describe a defined, reproducible platform for human embryonic stem cell differentiation.

Dr. E. Kingham, K. White, Prof. R. O. C. Oreffo

Bone and Joint Research Group

Centre for Human Development

Stem Cells \& Regeneration

Human Development and Health

Institute of Developmental Sciences

University of Southampton

Southampton, S016 6YD, UK

E-mail: roco@soton.ac.uk

Dr. N. Gadegaard

Division of Biomedical Engineering

School of Engineering

Rankine Building

University of Glasgow

Glasgow, G12 8LT, UK

Dr. M. J. Dalby

Centre for Cell Engineering

Institute of Molecular

Cell \& Systems Biology

College of Medical

Veterinary and Life Sciences

Joseph Black Building

University of Glasgow

Glasgow, G12 8QQ, UK

DOI: 10.1002/smll.201202340

\section{Introduction}

In contrast to adult stem cells, human embryonic stem cells (hESCs) exhibit infinite self-renewal and are pluripotent, differentiating to endodermal, ectodermal and mesodermal lineages. ${ }^{[1]}$ Maintenance of self-renewal, retention of pluripotency and differentiation requires specific intrinsic and extrinsic cues, ${ }^{[1]}$ the latter being provided by the chemical and physical surroundings. Thus the opportunity exists to manipulate the extrinsic hESC environment in order to obtain medically relevant cell types for stem cell biology, small molecule screening and therapeutic application.

Current orthopaedic research focuses on the use adult skeletal stem cells (SSCs), present within bone marrow, for regenerative therapies with the objective of producing osteogenic or chondrogenic cell types for transplantation and repair of bone or cartilage. ${ }^{[2]}$ However, in comparison to ESCs, SSCs are in limited supply (constituting 1 in 40 000-100 000 bone marrow cells), typically from older patients undergoing total hip replacement and display limited proliferation in vitro. Thus, ESCs may provide an alternative source of SSCs and SSC-derived cells in order to realise therapeutic applications. In vitro osteogenic differentiation protocols for mouse ESCs 
(mESCs) and hESCs have been reported ${ }^{[3-5]}$ however, the focus for these protocols has typically centred on chemical induction and/or embryoid body (EB) formation to induce differentiation.

We have recently described the manipulation of adult SSC differentiation in the absence of soluble factors using a defined nanotopography of $120 \mathrm{~nm}$ diameter pits in a near square arrangement. ${ }^{[6-8]}$ This work highlights an ability of the nanoscale physical environment alone to modulate stem cell fate. Additionally, nanopit depths of $100 \mathrm{~nm}$ but not $10 \mathrm{~nm}^{[9]}$ and nanotubes of $70 \mathrm{~nm}$ and $100 \mathrm{~nm}$ but not $30 \mathrm{~nm}$ in diameter ${ }^{[10]}$ were reported to favor SSC osteoblastic differentiation in the absence of osteogenic chemical cues indicating that scale, even within the nano range, is also important. Furthermore, the degree of order and disorder of nanotopography is elegantly demonstrated by the observations that a regular square arrangement of nanopits enhanced the stem cell state of SSCs ${ }^{[7]}$ whereas displacement of that order by $50 \mathrm{~nm}$ induced osteogenic differentiation. ${ }^{[6,8]}$ Osteogenic differentiation of mESCs and hESC has been described using nanoscale topography in the presence of soluble osteogenic factors and/or prior EB formation making it difficult to distinguish the effect of surface topography alone. ${ }^{[11]}$

SSCs and mESCs can be cultured on a wider range of surfaces compared to hESCs, while human pluripotent-derived cell types remain the Holy Grail for regenerative therapies. To this end, we have examined the potential for substrates lacking in topographical features, planar, and nanotopographical substrates displaying a near square pattern of nanopits (NSq50) to influence hESC differentiation in the absence of soluble, chemical induction factors and we have investigated the resulting changes in gene expression and epigenetic markers.

\section{Results}

\section{1. hESCs in the Presence or Absence of Topographical Cues Exhibit a Loss of Self- Renewal Marker Expression}

Differentiation of hESCs incubated on planar and NSq50 (Figure S1) substrates was assessed by immunofluorescent staining for self-renewal markers (Figure 1). On matrigel in MEF-conditioned media (CM), self-renewing hESCs displayed robust positive staining for OCT4 and SOX2 in nuclear localisations and TRA-1-60 and SSEA4 on the cell surface (Figure 1A) typical of self-renewing ESCs under routine culture. Following replacement of $\mathrm{CM}$ for hESC media lacking FGF for 3 days, a marked loss of expression of all self-renewal markers was observed (Figure 1A). hESCs seeded on planar substrates in basal media ( $\alpha$-MEM plus $10 \%$ serum) exhibited reduced staining for OCT4 and SOX2 after 2 days incubation however, TRA-1-60 and SSEA4 expression remained detectable (Figure 1A). On NSq50 substrates, hESCs also displayed reduced OCT4 and SOX2 expression following 2 days of culture although not as low as cells on planar substrates at this time point.

Increased incubation led to a further loss of self-renewal on the substrates. By day 4, expression of OCT-4, SOX2 and SSEA4 was further decreased in cells incubated on planar or NSq50 substrates. It is notable that TRA-1-60 remained detectable in localised areas (Figure 1A) particularly on planar substrates. Critically, expression of OCT4, SOX2 and TRA-1-60 were undetectable by day 14 on planar or NSq50 substrates (Figure 1B). SSEA4 expression was strong at day 14 on planar but on NSq50 substrates expression was negligible. Consistent with a loss of hESC self-renewal
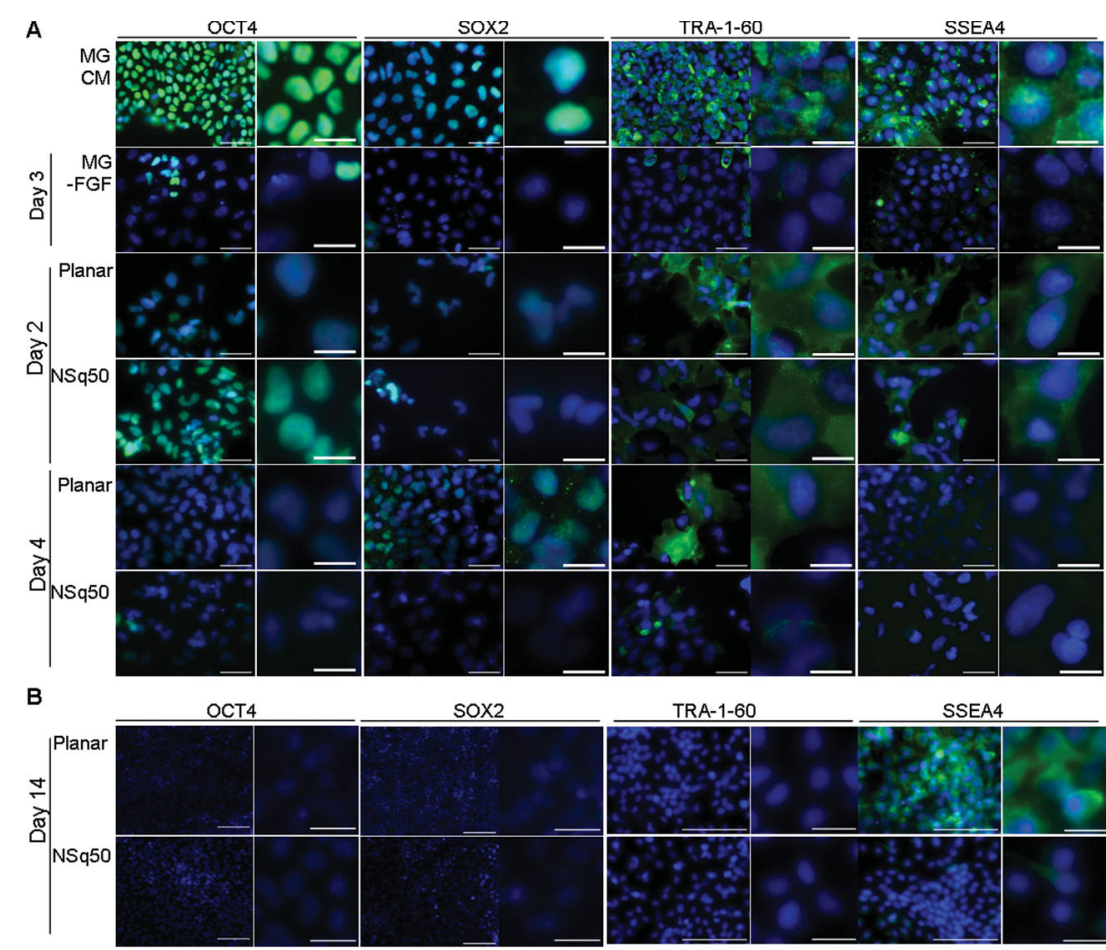

TRA-1-60

SSEA4

C
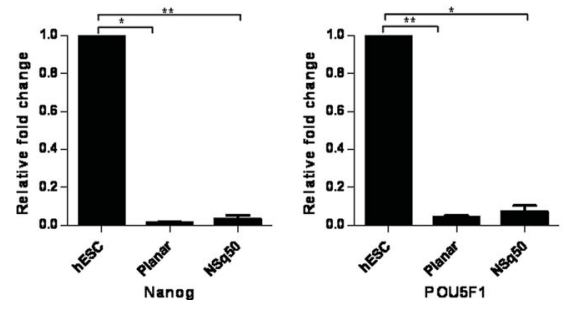

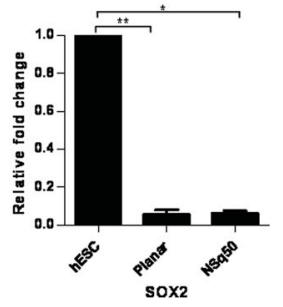

Figure 1. Self-renewal marker expression is reduced following incubation on planar or NSq50 substrates in basal media. hESCs seeded on matrigel in CM or in hESC medium lacking FGF were fixed after 3 days. hESCs seeded on planar or NSq50 nanotopographies in $\alpha-M E M$ media plus $10 \%$ serum were fixed after 2,4 (A, scale bars left panels $50 \mu \mathrm{M}$ and right panels $20 \mu \mathrm{M}), 7$ or 14 days (B, scale bars left panels $100 \mu \mathrm{M}$ and right panels $20 \mu \mathrm{M}$ ). Fixed cells were stained for OCT4, SOX2, TRA-1-60 or SSEA4 (green) and nuclei counterstained with DAPI (blue) (A and B). qPCR was used to assess mRNA expression of NANOG, POU5F1 and SOX2 following 14 days incubation on planar or NSq50 in basal media $(n=6)(C)$. 
protein expression, mRNA expression of NANOG, POU5F1 (encodes OCT4) and SOX2 was also significantly reduced for hESCs incubated on either planar or NSq50 substrates (Figure 1C). Importantly, comparison of live dead staining at days 4 and 12 revealed excellent viability with no discernible differences in the size or number of adhered colonies (Figure S2). Furthermore, after 12 days colonies had maintained excellent viability and colony size had increased to sub-confluent levels on the $2.5 \mathrm{~cm} \times 2.5 \mathrm{~cm}$ substrates displaying either planar or NSq50 topographies (Figure S2).

\subsection{Planar and NSq50 Substrates Do Not Direct Endodermal or Ectodermal Differentiation}

To investigate lineage specific differentiation, expression of a panel of early differentiation markers was assessed by qPCR and expression compared to day 14 EBs. At the mRNA level, the expression of visceral endodermal marker $\alpha$-fetoprotein (AFP) was significantly enhanced by differentiation on planar or NSq50 substrates compared to self-renewing hESCs (Figure 2A). However, AFP expression was variable and not significantly different from expression in day 14 EBs. Expression of endodermal marker SOX17 was low in hESCs and day 14 EBs (Figure 2A). Following differentiation on planar or NSq50 substrates, a significant reduction in expression of SOX17 was observed. Claudin6, another endodermal marker, was also expressed at a low level in hESCs with a significant reduction in expression following incubation on planar or NSq50 substrates and a greater reduction observed in day 14 EBs (Figure 2A).

Ectodermal marker SOX1, was not significantly enhanced in cells incubated on either planar or NSq50 substrates compared to hESCs (Figure 2B). However, a significant difference in SOX1 expression in cells on NSq50 compared to day 14 EBs was observed (Figure 2B). There is some debate as to whether neural differentiation is the default differentiation mode ${ }^{[12]}$ or that neural lineage differentiation is the fate of ESCs when extrinsic signals are minimal.[13] A significant enhancement in nestin expression was detected in day $14 \mathrm{EBs}$ compared to hESCs. In contrast, neuroectodermal differentiation was not evident on planar or NSq50 substrates since Nestin mRNA expression was almost undetectable in cells seeded on planar or NSq50 substrates (Figure 2B) and PAX6 expression was undetectable by qPCR in hESCs and hESCs seeded on planar or NSq50 for 14 days. Furthermore, cells incubated on planar or NSq50 substrates were negative for Nestin protein (Figure 2C). These expression profiles demonstrate that differentiation on planar or NSq50 substrates does not reproducibly induce endodermal or ectodermal cell types and for all genes investigated expression on nanotopographical substrates is distinct from the multiple lineage differentiation observed in EBs.

\subsection{Nanotopographical Cues Maintain Pan Stem Cell Marker Expression and Enhance Mesodermal and Osteogenic Marker Expression}

Expression of nucleostemin, a marker of ESCs, neural stem cells, bone marrow-derived SSCs and human cancer cells, ${ }^{[14]}$ was significantly reduced in cells on planar substrates but not significantly different in cells on NSq50 substrates compared to self-renewing hESCs (Figure 3A). Retention of nucleostemin expression after 14 days on NSq50 substrates, coupled with loss of self-renewal marker expression (Figure 1) and absence of neural marker expression (Figure 2) suggests differentiation towards cell types comparable to bone marrow stem cells on NSq50 substrates.

A statistically significant reduction in the expression of mesendodermal markers GSC (encodes goosecoid) (Figure 3B) and MIXL1 (Figure 3C) was observed in cells incubated on planar and NSq50 substrates compared to selfrenewing hESCs. In contrast, expression of the pan-mesodermal marker $\mathrm{T}$ (encodes brachyury) at day 14 was not significantly different (Figure 3D) potentially suggesting a rapid transition through the mesendodermal stage to mesodermal differentiation. BMP4 RNA expression was significantly enhanced (Figure 3E) and BMP4 protein expression was enhanced in cells incubated on planar and to a greater extent on NSq50 substrates (Figure 3F).

Given that SSCs are of mesodermal origin giving rise to osteogenic, chondrogenic and adipogenic lineages, this finding warranted investigation of SSC marker
Figure 2. Planar and NSq50 substrates do not direct endodermal or ectodermal differentiation. Expression of endodermal (A) and ectodermal (B) markers was assessed by qPCR for selfrenewing hESCs and hESCs seeded onto planar or NSq50 substrates in basal media for 14 days $(n=6)$. C) Following 14 days incubation, cells were fixed, stained for nestin (green) and counterstained with DAPI (blue). (Scale bars $50 \mu \mathrm{m}$ ). 


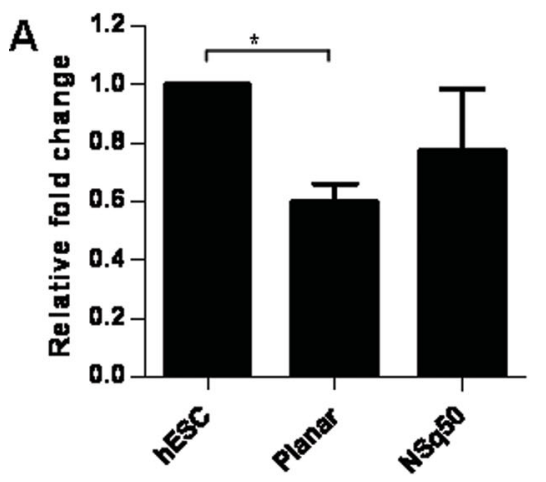

Nucle oste $\mathrm{m}$ in
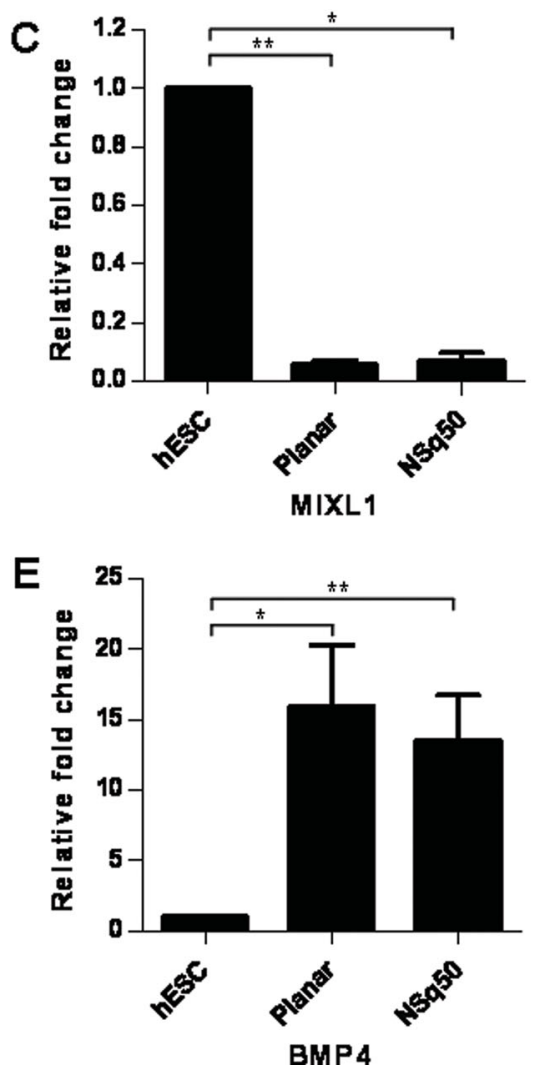

$\mathbf{F}$
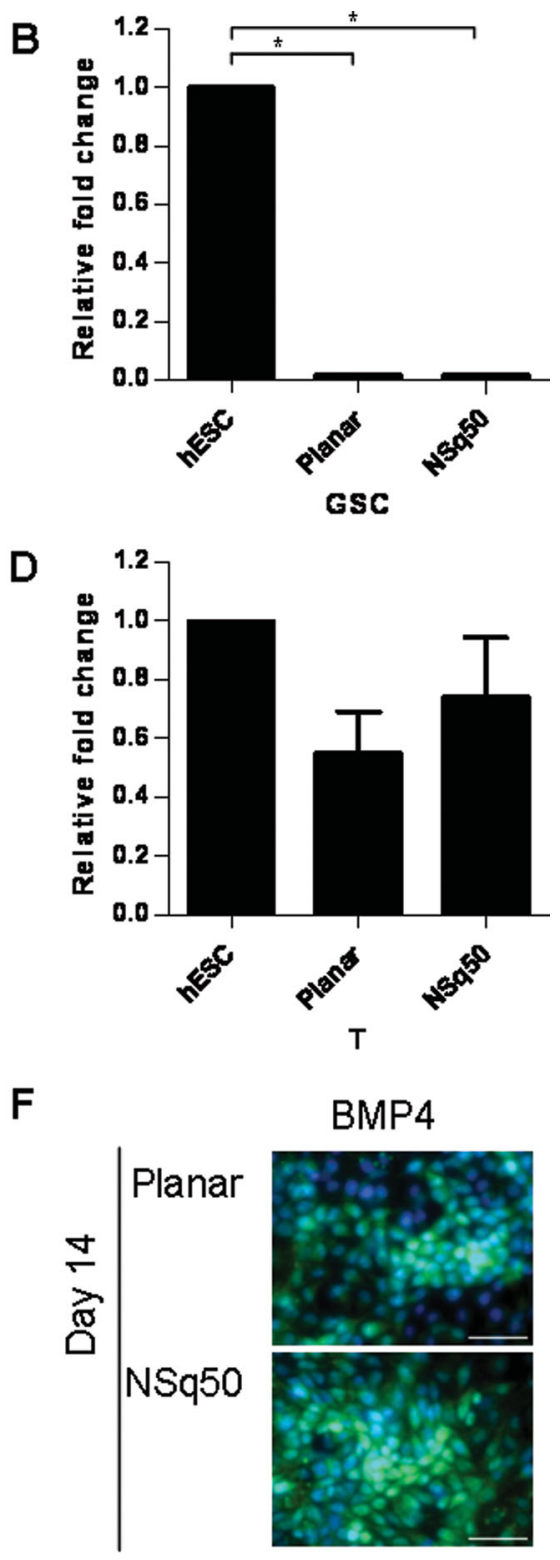

Figure 3. NSq50 maintains pan-stem cell marker expression and enhances expression of mesodermal marker. BMP4 Expression of nucleostemin $(n=6)(A)$, GSC $(n-5)(B)$, MIXL1 $(n=$ 5) $(C), T(n=6)(D)$ and BMP4 $(n=6)(E)$, was assessed by qPCR for hESCs and hESCs seeded onto planar or NSq50 in basal media for 14 days. Cells were fixed after 14 days incubation and stained for BMP4 (F) (green) and counterstained with DAPI (blue). (Scale bars $50 \mu \mathrm{m}$ ).

14, STRO1 expression was similar to that observed on day 7 for planar substrates but slightly reduced for NSq50 substrates (Figure 4B). CD44 is a marker of bonemarrow derived mesenchymal stem cells (MSCs). ${ }^{[17]}$ CD44 expression was low in hESCs, enhanced following differentiation on planar substrates and enhanced to a greater extent on NSq50 substrates on day 7 (Figure 4B). CD44 expression on planar substrates was further enhanced at day 14. CD44 expression on NSq50 substrates had reduced slightly by day 14 compared to day 7. Thus, cells differentiating on NSq50 substrates appeared to strongly gain and then exhibit a reduction in expression of CD44 and STRO1 protein. One possible explanation for this is that NSq50 augments or extends the differentiation of hESCs beyond that achieved by planar substrates, possibly towards SSC-like cell types. At day 14, CD44 mRNA expression was significantly enhanced in cells on NSq50 substrates although not in cells on planar substrates compared to hESCs (Figure 4C) indicating an overall enhancement in the expression of CD44.

Consistent with temporal changes in CD44 and STRO1 expression on NSq50 substrates, later markers of primitive human stromal cell differentiation were detected at day 14. Collagen type I (bonespecific) was enhanced significantly in cells incubated on planar substrates and to a greater extent in cells incubated on NSq50 substrates (Figure 4D). Expression of collagen type I protein by cells differentiated on planar and NSq50 for 14 days was confirmed by immunofluorescent staining (Figure 4E). Collagen type II (cartilage-specific) was significantly enhanced on either planar or NSq50 substrates (Figure 4F). RUNX2, a critical transcription factor for induction of osteogenic differentiation, ${ }^{[18]}$ and osteonectin, expression. CD63, a marker of primitive human marrow stromal cells ${ }^{[15]}$ was significantly enhanced in cells incubated on NSq50 substrates but not significantly different in cells incubated on planar substrates (Figure 4A). Furthermore, ALCAM, a marker of bone marrow stromal cells and osteoblastic cell lines was significantly enhanced in cells incubated on planar with greater expression observed on NSq50 substrates (Figure 4A).

STRO1 is reported as a reliable marker of a bone marrow-derived SSC population and osteogenic progenitor cells. ${ }^{[15-16]}$ Low levels of STRO1 expression was detectable in hESCs (Figure 4B). STRO1 expression was enhanced following 7 days on either planar or NSq50 substrates. On day a bone-specific protein forming complexes with collagen, ${ }^{[19]}$ were significantly enhanced on planar and NSq50 substrates (Figure 4F). Conversely, PPAR $\gamma$, reported to be involved in early mesodermal induction and adipogenesis ${ }^{[20]}$ was undetectable by qPCR in hESCs or hESCs differentiated on planar or NSq50 substrates for 14 days.

Taken together, these observations indicate directeddifferentiation of hESCs towards mesenchymal cell types by either planar or NSq50 substrates in the absence of chemical induction factors. However, differences in the expression profiles of the markers investigated (namely Nucleostemin, CD63, CD44 and STRO1) indicate that nanotopographical cues provided by NSq50 substrates influence cell fate in a 
A
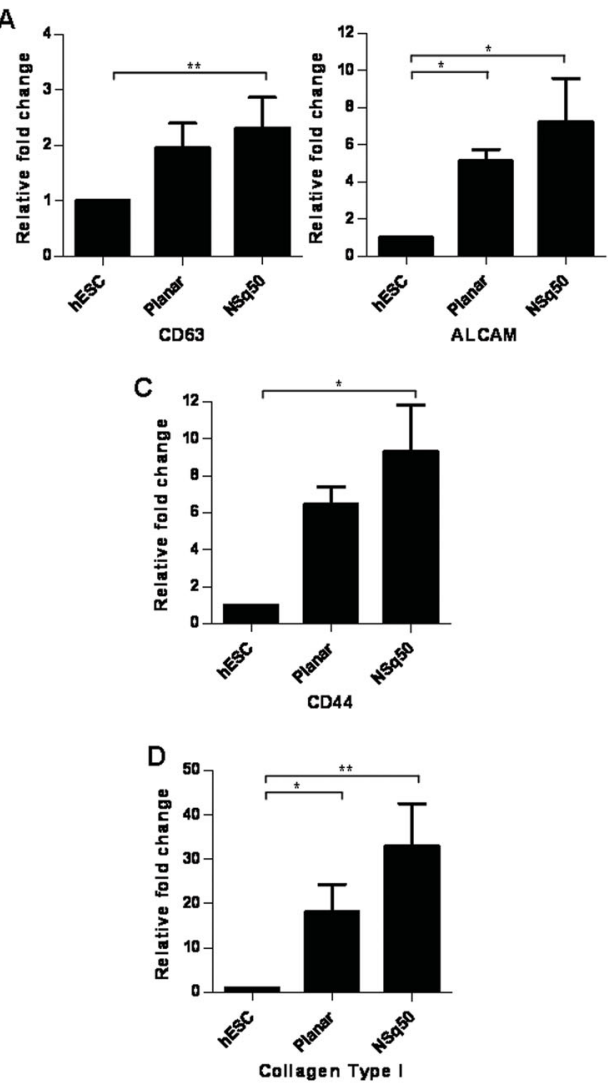

B

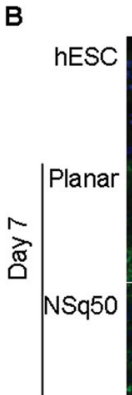

STRO1

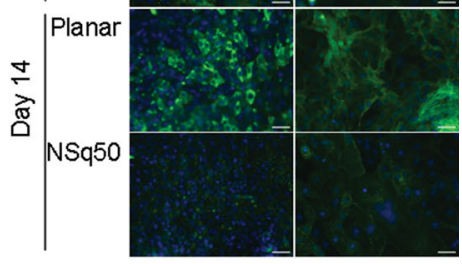

E

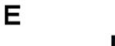

Collagen Type I

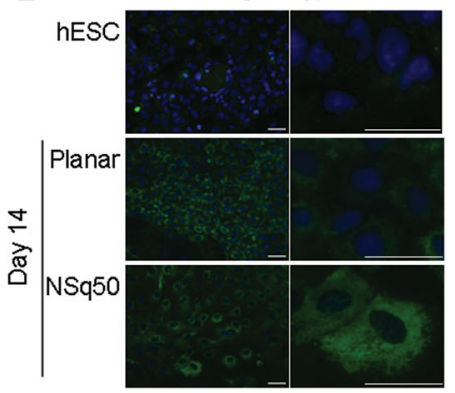

$\mathbf{F}$

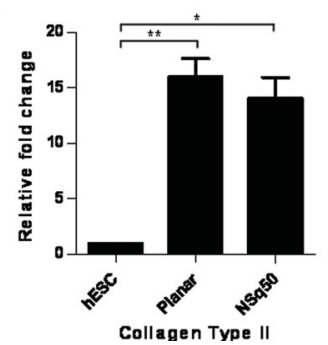

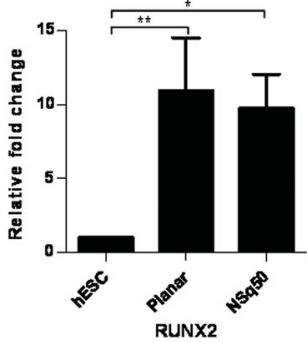

RUNX2

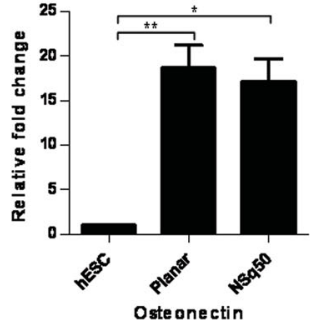

Osteonectin

Figure 4. NSq50 enhances expression of skeletal stem cell and osteogenic progenitor markers. Expression of CD63, ALCAM ( $n=5)(A)$, CD44 $(n=3)(C)$, collagen type I (D), collagen type II, RUNX2 and osteonectin $(n=6)(F)$ was assessed by qPCR for hESCs and hESCs seeded onto planar or NSq50 in basal media for 14 days. Cells were fixed after 7 and 14 days incubation, stained for STR01, CD44 (B) or collagen type I (E) (green) and counterstained with DAPI (blue). (Scale bars $50 \mu \mathrm{m}$ ).

manner that differs from planar substrates. NSq50 substrates augment hESC mesenchymal differentiation to produce cells expressing SSC markers.

In contrast to hESCs, EBs were less sensitive to nanotopographical cues for mesoderm/mesenchymal differentiation. Despite adherence and outgrowth of day 4 EBs on planar and NSq50 substrates (Figure S4A), no indication of directed differentiation by planar or NSq50 substrates was observed. EB-derived cells exhibited expression of markers representing all three germ layers and no apparent trends in germ layer marker expression (Figure S4B). Thus, following EB formation, cells appeared to be less responsive to both a lack of topography and to nanotopography-induced mesodermal differentiation. tein expression (Figure 6A).

\author{
2.4. Nanotopographical Cues Induce \\ Morphology and Changes in Adhesion \\ Protein Expression Similar to Those \\ Observed During Epithelial to Mesen- \\ chymal Transition
}

Cell morphology was investigated by bright field microscopy at days 2, 5, 9, and 12 after seeding (Figure 5). Cell morphology was similar for hESCs seeded on planar or NSq50 substrates on days 2, 5, and 9. However at day 12, the cell population on planar substrates displayed a more heterogeneous appearance with some overlapping cells. On NSq50 substrates, cells were arranged in a monolayer with a high degree of cell-cell contact surface area (Figure 5). This epitheliallike cell morphology observed on NSq50 substrates was similar to that reported by Chen and co-workers using TGF- $\beta$ inhibition to induce mesenchymal differentiation of ESCs. ${ }^{[21]}$

Epithelial to mesenchymal transition (reviewed by Lee et al) ${ }^{[22]}$ occurs during gastrulation when mesodermal and endodermal cell populations are formed. Reciprocal expressional changes in E-cadherin and $\mathrm{N}$-cadherin are reported to be a hallmark of this process in pluripotent cells. ${ }^{[1,23]}$ Expression of E-cadherin protein was detected in cells cultured on planar and NSq50 substrates for 5 days (Figure 6A). After 10 days expression was reduced and after 14 days protein expression was almost undetectable on planar or NSq50 substrates (Figure 6A). E-cadherin mRNA expression was not significantly different in cells incubated for 14 days on planar substrates (Figure 6B). However, a statistically significant reduction in E-cadherin mRNA expression was observed in cells differentiated on NSq50 substrates for 14 days compared to self-renewing hESCs (Figure 6B) correlating with pro-

In contrast, expression of $\mathrm{N}$-cadherin was detectable at day 5 although to a greater extent in cells seeded on NSq50 substrates (Figure 6C). On either substrate N-cadherin expression was significantly reduced on day 10 compared to day 5. For cells on planar substrates, expression remained low on day 14 but on NSq50 substrates expression was significantly enhanced on day 14 . The observation of a loss of E-cadherin expression on either substrate is consistent with differentiation of hESCs. However, cells on NSq50 substrates also exhibited an enhancement in $\mathrm{N}$-cadherin expression which, taken together with reduced E-cadherin expression, is consistent with the epithelial to mesenchymal transition process in ESCs. ${ }^{[21,23]}$ 


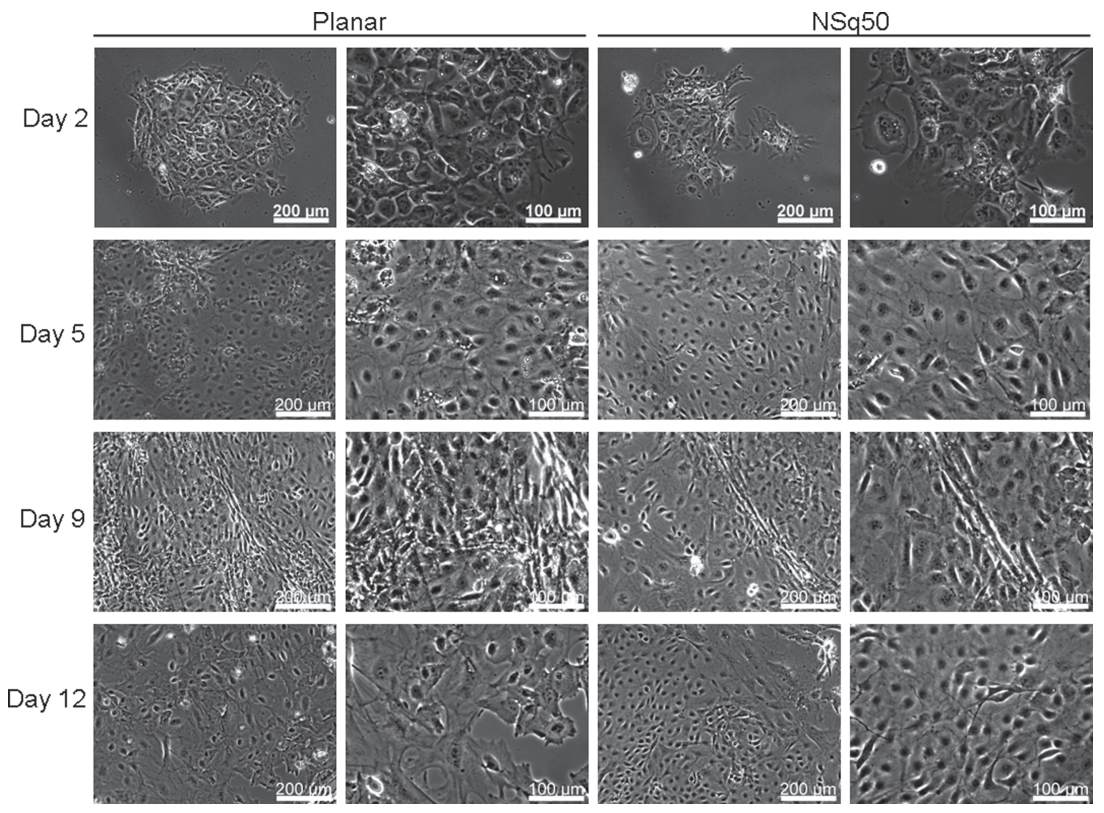

Figure 5. hESC morphologies during differentiation on planar and NSq50 substrates. hESCS were seeded onto planar and NSq50 substrates and incubated for 12 days. Bright field microscopy images were taken on days 2, 5, 9, and 12. (Scale bars $100 \mu \mathrm{m}$ or $200 \mu \mathrm{m}$ as indicated.)

The zinc finger transcription factors and repressors of E-cadherin transcription SLUG ${ }^{[24-25]}$ and SNAIL ${ }^{[26]}$ are reported induce epithelial to mesenchymal transition. ${ }^{[24,27]}$ Furthermore, the repression of SLUG in the developing embryo prevents primitive streak mesoderm formation ${ }^{[28]}$ while SLUG/SNAIL expression has previously been reported in ESC differentiation during epithelial to mesenchymal transition. ${ }^{[29]}$ hESCs incubated on planar and NSq50 for 5 days displayed a low level of nuclear SLUG/SNAIL expression (Figure 6D). By day 10, expression of SLUG/SNAIL was enhanced in cells on either surface. Localisation of SLUG/ SNAIL remained predominantly nuclear for cells on planar substrates but both nuclear and cytoplasmic staining was observed in cells on NSq50 substrates. At day 14, expression of SLUG/SNAIL was detected within the nuclei and at low levels in the cytoplasm of cells incubated on planar substrates. Expression SLUG/SNAIL was enhanced to a greater extent in cells incubated on NSq50 substrates for 14 days with both nuclear and cytoplasmic localisation (Figure 6D).

\subsection{NSq50 Induces a 'Mesenchymal Stem Cell'-Like Cell Phenotype}

To fully characterise the differentiated cell types, MSC PCR arrays with 84 mesenchymal-focused genes, 5 house-keeping genes and 7 internal controls were employed. A hierarchical clustering heatmap depicts the differences in gene expression (Figure 7A). Notably, hESCs differentiated on planar and NSq50 substrates are distinct from self-renewing hESCs. Moreover, differential gene expression between cells incubated on planar substrates or on NSq50 substrates was evident (Figure 7A). PCR Array data highlighted 10 genes down-regulated at least 2 fold (i.e. fold regulation <-2) following differentiation on planar or NSq50, in comparison to selfrenewing hESCs. Self-renewal markers FGF2, POU5F1, SOX2 and REX1 were significantly down-regulated by at least 2 fold following differentiation on planar or NSq50 (Figure 7B). Incubation on planar resulted in 35 genes up-regulated greater than 2 fold whilst differentiation on NSq50 induced up-regulation greater than 2 fold for 36 genes, with 32 genes mutually up-regulated. The filtered data set identified eleven genes up-regulated to a greater extent on NSq50 than on planar when compared to self-renewing hESCs (Figure 7C) of which eight genes are specifically expressed by or associated with MSCs, two are associated with osteogenesis and one associated with mesodermal differentiation (Table S3). Furthermore, enhanced expression of MSC and osteogenic lineage markers (broadly and namely CD44 and collagen type I) by NSq50 substrates compared to planar substrates identified by PCR array is in agreement with our qPCR and immunofluorescent staining observations (Figure 4).

Differential expression (identified by normalising NSq50 PCR array data to planar data) (Figure 7D) highlighted two genes up-regulated greater than 1.5 fold on NSq50; Hepatocyte nuclear factor 1 alpha (HNF1A), is reported to enhance MSC proliferation downstream of Wnt signalling and to delay osteogenic maturation, ${ }^{[30]}$ and protein tyrosine phosphatase, receptor type C (PTPRC), a stromal cell marker, were upregulated 1.96 and 1.72 fold respectively in cells incubated on NSq50 (Figure 7D). Additionally, 10 genes were downregulated greater than 1.5 fold in cells incubated on NSq50 (Figure 7D); including PPAR $\gamma$, an inducer of adipogenic differentiation, ${ }^{[20]}$ which was undetectable by the less sensitive method of qPCR (Section 3.3). Interestingly, siRNA-mediated knockdown of PPAR $\gamma$ has been reported to enhance osteogenic marker expression in mESCs. ${ }^{[31]}$ Differential gene expression highlights the influence of discrete nanopit pattern displayed by NSq50 substrates on directed differentiation of hESCs.

\subsection{Methylation Status of POU $5 \mathrm{~F}_{1}$ and Osteocalcin Promoters Following Differentiation on Planar and NSq50}

Following significant expression profile changes in cells incubated on planar and NSq50, changes at an epigenetic level were determined. Global methylation of hESCs and following incubation on planar or NSq50 substrates was not statistically different (Figure S3). POU5F1 contains a number of $\mathrm{CpG}$ sites upstream and downstream of the transcription initiation site, at which methylation corresponds to reduced transcription and reduced expression of OCT4. ${ }^{[32]}$ In general, 
A
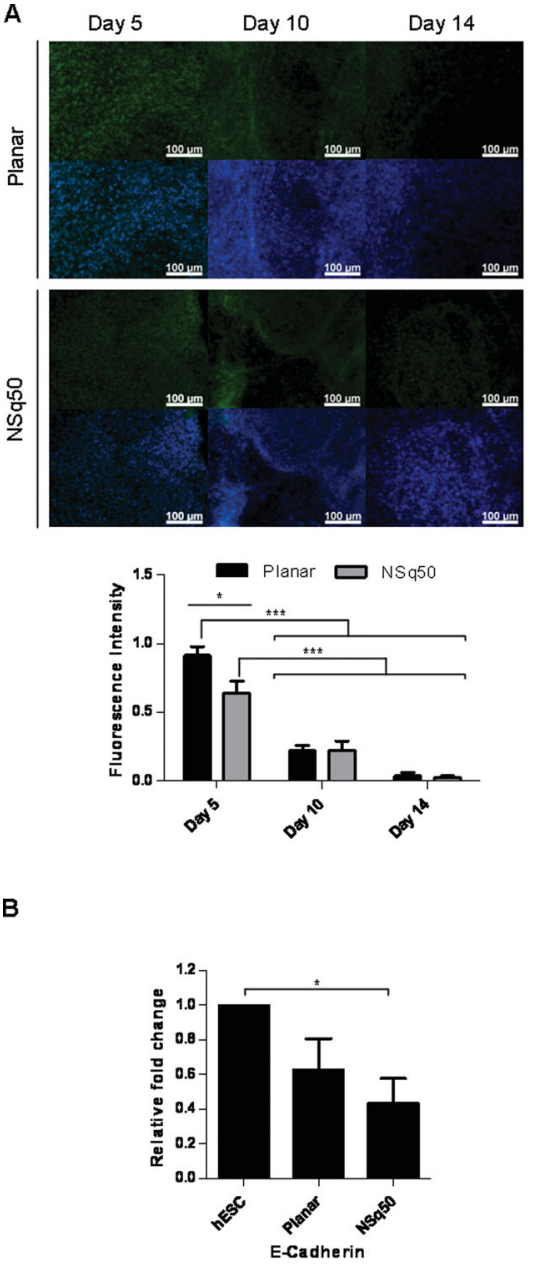

C
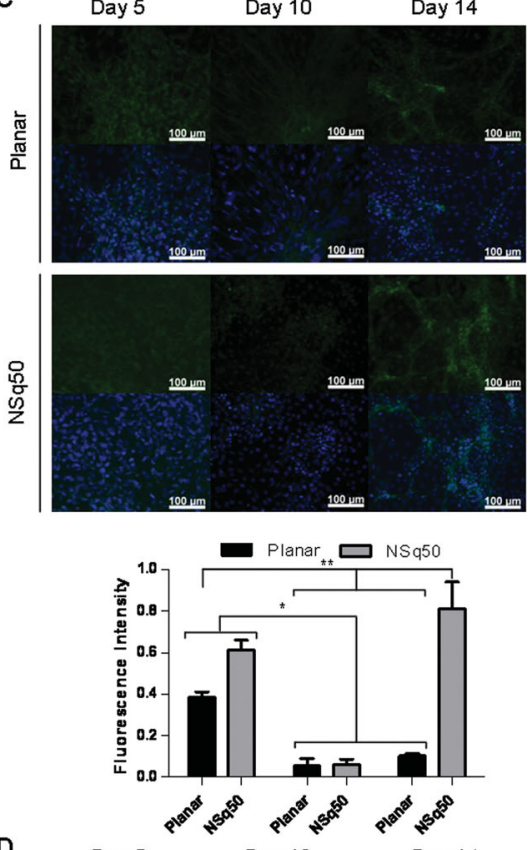

D

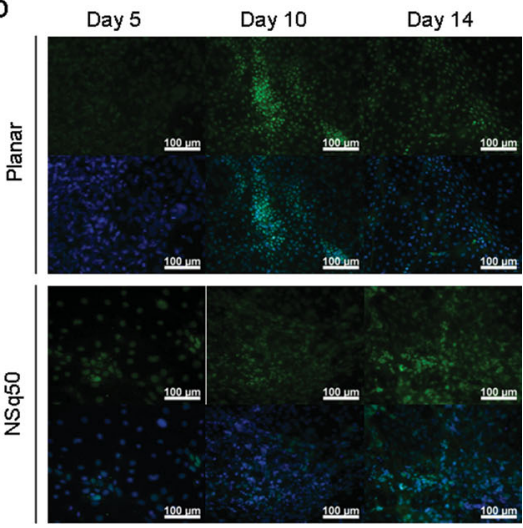

Figure 6. Reciprocal expression changes of E-cadherin and $\mathrm{N}$-cadherin during planar and NSq50 substrates-induced mesenchymal differentiation Cells were fixed after 5, 11, and 14 days incubation on planar or NSq50 substrates, stained for E-cadherin (A), N-cadherin (C) or SLUG/SNAIL (D) shown in green in upper panels. Middle panels show merged images of staining and DAPI counterstain (blue). (Scale bars $100 \mu \mathrm{m}$ ). Fluorescence intensity of $\mathrm{E}$-cadherin and $\mathrm{N}$-cadherin immunostaining was quantified and represented graphically as a mean $(\mathrm{n}=3)$ with standard deviation (A and $\mathrm{C}$ lower panels). Expression of E-cadherin (B) was assessed by qPCR for hESCs and hESCs seeded onto planar or NSq50 in basal media for 14 days.

hypomethylation of $\mathrm{CpG}$ sites in POU5F1 promoter region (Figure 8A) was detected in hESCs which was enhanced following removal of $\mathrm{CM}$ to induce differentiation (Figure 8B), consistent with a loss of OCT4 expression (Figure 1). Specifically, for CpG sites 109 base pairs upstream and 4, 29, 39, 79, and 87 base pairs downstream of the transcription initiation site, incubation on planar or NSq50 substrates induced a significant increase in methylation compared to self-renewing hESCs (Figure 8B) correlating with a loss of OCT4 expression (Figure 1). Moreover, methylation levels in nanotopographydifferentiated hESCs were not statistically different from levels in cells induced to differentiate by withdrawal of CM.

Following observation of enhanced SSC and early osteoinductive marker expression including RUNX2 (Figure 4), cifically, retention of Nucleostemin, a significant enhancement in CD63 and ALCAM, a significant reduction in E-cadherin expression, temporal expression of CD44 and STRO1, upregulation of markers associated with stromal/mesenchymal cell types and down-regulation of markers associated with non-stromal/mesenchymal cell types were observed in cells on NSq50 but not planar substrates.

Previous reports detailing mESC-derived osteogenic cells via cell aggregation or bioreactor-differentiation protocols indicate the maintenance of a sub-population of teratomaforming undifferentiated ESCs. ${ }^{[3,39]}$ Here, protein expression of OCT4, SOX2 and TRA-1-60 was undetectable after 14 days on NSq50. Naturally, the complete absence of undifferentiated cells following nanotopographical differentiation 
A

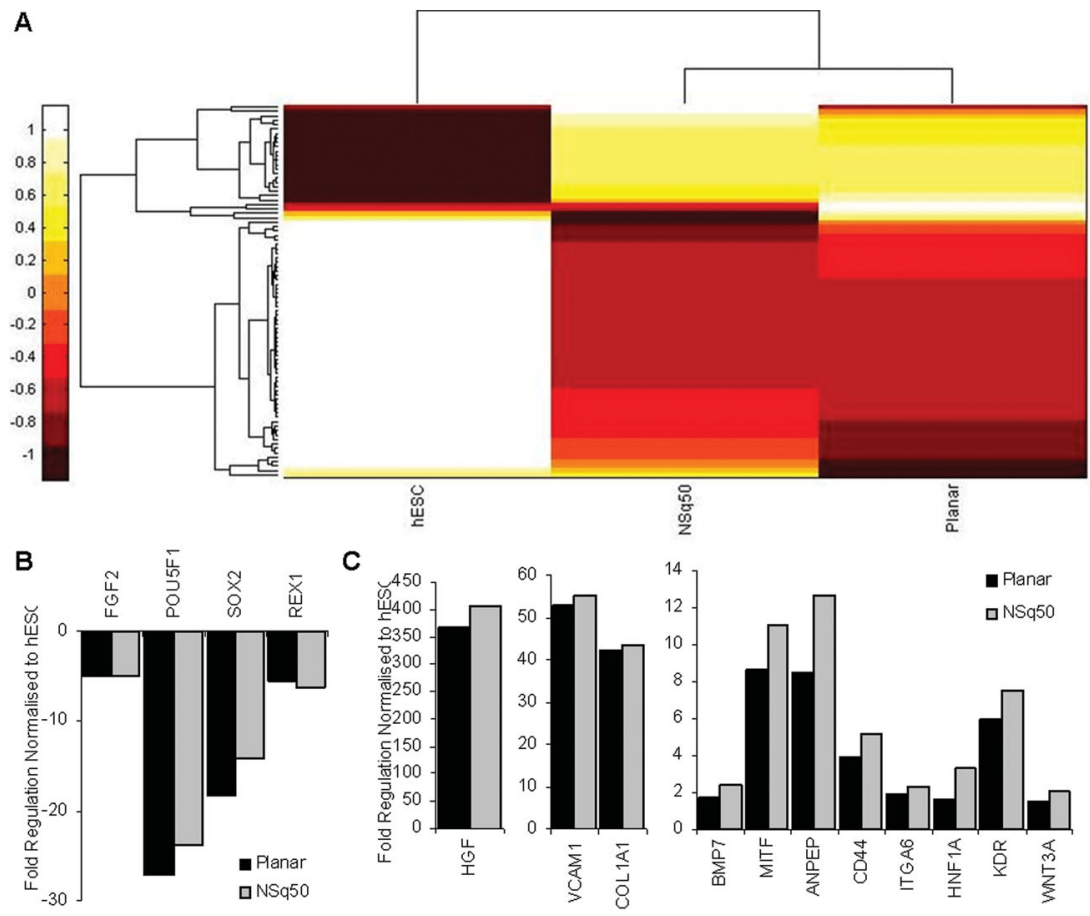

D

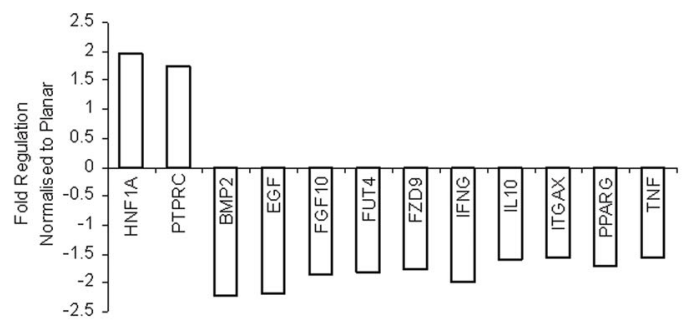

Figure 7. Gene expression profile of hESCs cultured on planar and NSq50. Gene expression was profiled using MSC RT2 PCR array for self-renewing hESCs $(n=1)$ and for hESCs differentiated for 14 days on planar $(n=3)$ or NSq50 $(n=3)$ substrates. Cluster analysis is represented as a heat map where low to high expression is represented by dark red to white (A). PCR array data was analysed online with RT2 Profiler PCR Array Data Analysis (SA Biosciences) tool and normalised to self-renewing hESCs (B and C) or to planar (D). B Self-renewal marker genes down-regulated greater than 2 fold following culture on planar or NSq50. (C) Genes up-regulated greater than 2 fold were filtered for genes up-regulated more on NSq50 than on planar. (D) Genes differentially expressed following culture on NSq50 compared to planar. cell lines towards mesodermal differentiation. ${ }^{[43]}$ Importantly, we found no evidence of a spontaneous mesodermal propensity of this cell line since expression of markers representing all three germ layers were detectable in EBs indicating multiple lineage differentiation capacity. Induction of differentiation by EB formation prior to seeding reduced the susceptibility to influence by either the absence or presence of nanotopographical cues. In part, this can be attributed to cell-surface contact since EBs exhibited outgrowth on all surfaces but had already formed cell-cell contacts thus reducing cell-substrate contact upon seeding consistent with the procedure of optimising cell-surface contact in order to maximise cell-surface influence over and above cell-cell contact. ${ }^{[35]}$ It has previously been reported that EB formation prior to chemically-induced osteogenic differentiation is not essential and may, in fact, limit osteogenic potential. ${ }^{[5,44]}$ Moreover, in light of surface stiffness ${ }^{[35]}$ and cell shape ${ }^{[36]}$ coupling to cell fate, Chowdhury et al. demonstrated that undifferentiated mESCs were seven times softer (higher cell deformation in response to the same applied stress) and exhibited greater spreading in response to local cyclic stress compared to differentiated mESCs. ${ }^{[45]} \mathrm{A}$ reduction in the softness of cells subsequent to differentiation by EB formation may prevent nanotopographical influence on cell shape and inhibit nanotopographical-directed differentiation.

Sox17 expression is required for endoderm specification in mice but is not expressed in mesodermal, ectodermal or trophectodermal lineages. ${ }^{[46]}$ D'Amour et al., reported elevated expression of SOX17, would need to be confirmed by in vivo studies. SSEA4 was detected in hESCs differentiated on planar substrates and at very low levels in hESCs differentiated on NSq50 substrates. SSEA4 expression was reported in bone marrow aspirate cells, ${ }^{[40]}$ human first-trimester fetal $\mathrm{MSCs}^{[41]}$ and circulating SSEA4+ cells of bone marrow origin were reported to be detected in patients with acute myocardial infarction. ${ }^{[42]}$ These reports suggest that our observation of SSEA4 expression in hESC-derived mesenchymal/stromal cells supports the mesenchymal phenotype of these cells.

Neural differentiation of hESCs is reported as the default lineage ${ }^{[12]}$ or the lineage favoured in the absence of chemical cues in the culture media. ${ }^{[13]}$ Expression of neural markers PAX6 and nestin were undetectable at the mRNA and protein levels respectively in hESCs incubated for 14 days on planar or NSq50 substrates. However, this cell line has capactiy for neural differentiation evidenced by the expression of Pax6 and nestin in EBs. Furthermore, others report a bias of some
GSC and MIXL1 in activin A-induced hESC differentiation to definitive endoderm. ${ }^{[47]}$ In addition, Claudin6 is reported to be expressed in derivatives of definitive endoderm. ${ }^{[48]} \mathrm{We}$ observed no enhancement in the expression of these markers in cells incubated on planar or NSq50 substrates suggesting that the population of cells is not endodermal. Brachyury is expressed in mesendoderm and mesodermal lineages and is reportedly required for mesoderm induction. ${ }^{[49]}$ In contrast to GSC and MIXL1, expression of T, the gene encoding brachyury, was not significantly different in cells differentiated on planar or NSq50 substrates. In this instance we postulate that a small number of cells had retained $\mathrm{T}$ expression at day 14 while most cells had differentiated past this stage.

Cells incubated on NSq50 substrates displayed enhanced expression of markers of mesenchymal, stromal and early osteogenic progenitors above that detected in cells on planar substrates in the same basal media. Up-regulated expression of E-cadherin transcriptional repressors SLUG/SNAIL 
A

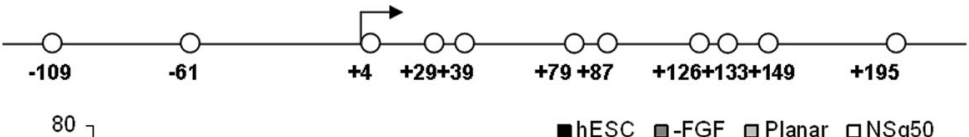

B
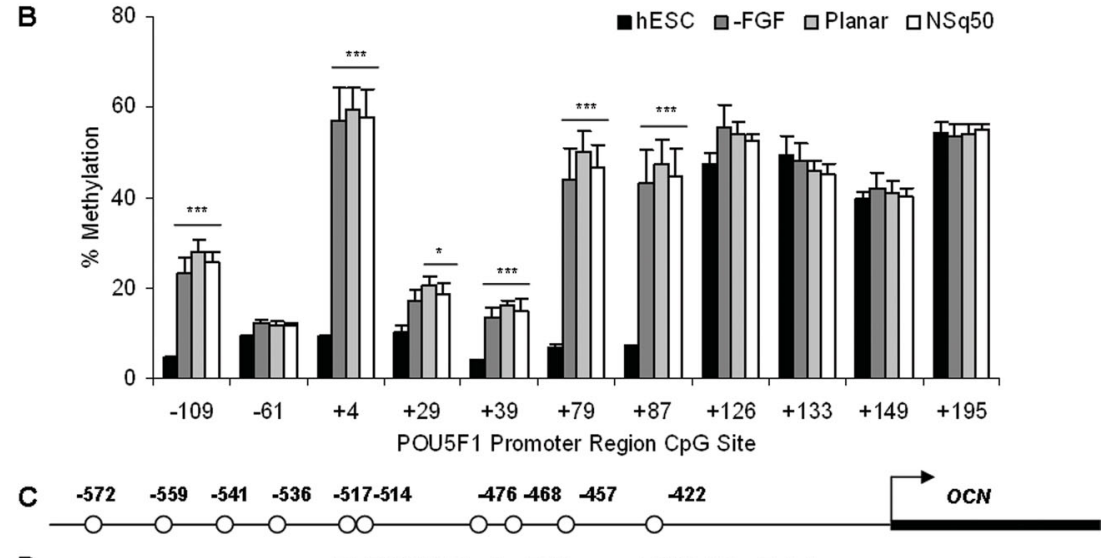

D

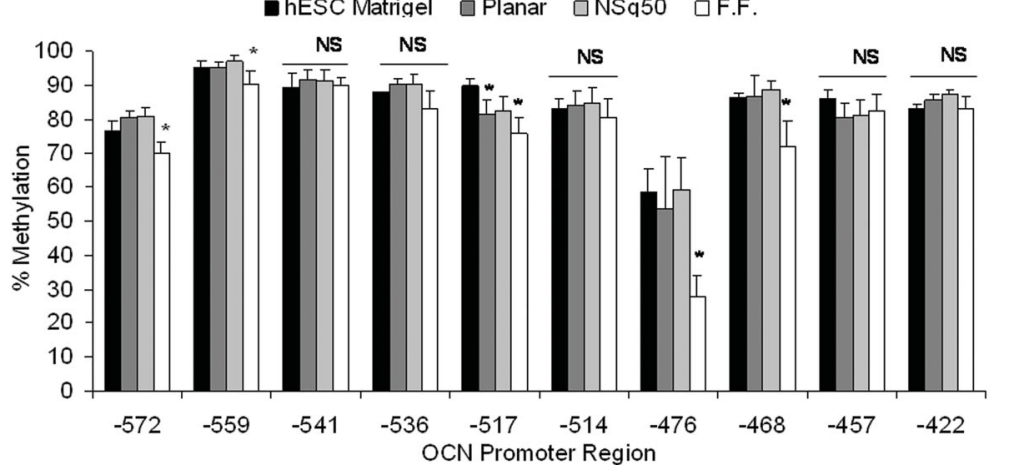

Figure 8. Methylation status of POU5F1 and osteocalcin promoter regions following differentiation on planar and NSq50 POU5F1 (A) and osteocalcin (C) promoter regions were analysed for $\mathrm{CpG}$ methylation by pyrosequencing. The percentage methylation of the POU5F1 promoter region $\mathrm{CpG}$ sites for self-renewing $\mathrm{hESCs}$, cells incubated for 14 days on tissue culture plastic in hESC medium lacking FGF ('-FGF'), on planar or NSq50 substrates in basal medium is shown $(n=3)(B)$. The percentage methylation of the osteocalcin promoter region for self-renewing hESCs $(n=4)$, cells incubated for 14 days on planar or NSq50 substrates in basal media $(n=4)$ and fetal femur explants ('F.F.') $(n=5)$ is shown (D).

and localization within the nucleus coupled with a temporal down-regulation of E-cadherin and reciprocal up-regulation of $\mathrm{N}$-cadherin protein expression following planar or NSq50 substrate differentiation of hESCs indicates an epithelial to mesenchymal transition during differentiation on substrates. Expression of SLUG/SNAIL was greatest in cells incubated in NSq50 substrates correlating with our observations of epithelial-like cell morphologies on NSq50 but not planar. Evidence of this transition further supports mesenchymal lineage differentiation.

The ability of nanotopographical cues to modulate cell types within this lineage has previously been reported. We have formerly described osteogenic differentiation of SSCs by NSq50 substrates ${ }^{[6]}$ and retention of MSC stemness by a square arrangement of nanopits. ${ }^{[7]}$ The nanoscale architecture of MSC osteoid was reported to inform skeletal tissue generation. ${ }^{[50]}$ Moreover, osteoblast or adipocyte formation was reported to be modulated by spatial fibronectin distribution which induced altered MSC tension, highlighting regulation by geometric cues. ${ }^{[37]}$ The importance of matrix stiffness in myogenic and osteogenic MSC differentiation has also been reported. ${ }^{[35]}$ However, hESCs represent a more challenging cell type yet potentially more diverse in prospects for regenerative medicine and tissue engineering. Despite the apparent absence of a completely flat surface in vivo we have demonstrated a cellular response to planar substrates and by implementing a nanopit arrangement on substrates of the same format, chemical and physical composition we have demonstrated an augmentation of this cellular response. Moreover, our studies were conducted in the absence of soluble chemical induction factors and without applying a functionalized surface coating to substrates. These steps have isolated, as far as possible, nanotopographical cues from soluble chemical, insoluble chemical and surface stiffness factors. Clues to a mechanism linking nanotopographical sensing might be found in tension, nanomechanical and cell spreading studies which heavily implicate cytoskeletal components. A conformational change in vimentin is suggested to protect the cytoplasm from large deformation $^{[51]}$ while vimentin phosphorylation by Rho-associated kinase mediates the cellular shape changes of neurite retraction. ${ }^{[52]}$ In mESCs, stress-induced spreading was reported to be dependent on myosin light chain phosphorylation and the expression of cdc42 and Src. ${ }^{[45]}$ Chen and co-workers demonstrated that integrin-mediated focal adhesions and E-cadherin regulate hESC sensing of topographical cues and that, on a smooth glass surface, self-renewal marker OCT3/4 positive cells have a greater spread area than OCT3/4 negative cells. ${ }^{[53]}$ Moreover, focal adhesion formation and E-cadherin have recently been implicated in both cytoskeleton contractility and mechanoresponsive differentiation. ${ }^{[54]}$

\section{Conclusion}

We have illustrated the potential of a planar substrate lacking in topographical cues, to modulate hESC differentiation towards mesodermal lineages. Substrates displaying a near square arrangement of nanopits provide enhance directed mesodermal differentiation of hESCs with a greater efficiency than planar substrates. The mechanisms by which nanotopography might induce mesodermal differentiation likely include expressional changes in adhesion proteins and regulators of epithelial to mesenchymal transition. In addition, the mechanism involves methylation changes akin to chemically induced induction. Nanotopography-induced differentiation provides a non-intrusive, facile approach for hESC culture in the absence of chemical cues. Cells produced by 
nanotopography-induced differentiation have wide applications in stem cell biology research, small molecule screening and therapeutic applications in regenerative medicine and tissue engineering.

\section{Experimental Section}

Materials: Nanotopography substrates were made as previously detailed. ${ }^{[6]}$ In brief, silicon masters of either planar or near square pattern of nanopits were used to imprint polycarbonate by hot-embossing. Nanopit dimensions of $120 \mathrm{~nm}$ diameter and $100 \mathrm{~nm}$ deep were positioned in a near square geometry with an average centre spacing of $300 \mathrm{~nm} \pm 50 \mathrm{~nm}$ from true centre (NSq50) (Figure S1).

Fetal Femur Explant Preparation: All human fetal bones used in this study were obtained, following informed written consent, after termination of pregnancy with ethical approval from the Southampton \& South West Hampshire Local Research Ethics Committee. Human fetal femurs and long bones were dissected and surrounding soft tissue removed. Fetal bones were sliced into small pieces and incubated with $1 \mathrm{mg} / \mathrm{ml}$ collagenase B overnight. The cell suspension was filtered to remove debris and cells pelleted under centrifugation. The cell pellet was lysed and RNA/DNA isolated using AllPrep RNA/DNA Kit (Qiagen).

Cell Culture: Maintenance of HUES-7 hESCs (D. Melton, Howard Hughes Medical Institute/Harvard University) was conducted on $\gamma$-irradiated MEFs in Knockout DMEM (Invitrogen) supplemented with 10\% knockout serum replacement (Invitrogen), L-glutamine (1 mM) (Invitrogen), $\beta$-mercaptoethanol (50 $\mu \mathrm{M}$ ) (Sigma), nonessential amino acids (0.1 mM) (Invitrogen), basic FGF (10 ng/mL) (Peprotech Ltd, London, UK) and penicillin/streptomycin (100 $\mu \mathrm{g} /$ $\mathrm{mL}$ ) (Invitrogen). Subsequent maintenance of HUES-7 on matrigelcoated (BD Biosciences) tissue culture plastic with $24 \mathrm{~h}$ MEFconditioned medium (C. M.) followed. All cell culture was conducted at $37^{\circ} \mathrm{C}$ in atmospheric $(-20 \%)$ oxygen.

Seeding Nanotopography Substrates with ESCs: Prior to seeding, nanotopography surfaces were incubated in antibiotic/antimycotic solution (Sigma) overnight, washed twice with PBS followed by one wash with medium. ESCs were dissociated from matrigel-coated tissue culture plastic by incubating with $1 \mathrm{mg} / \mathrm{mL}$ collagenase (Invitrogen) for $5 \mathrm{~min}$ at $37^{\circ} \mathrm{C}$ followed by manual cell scraping. ESCs were seeded onto nanotopography surfaces by 1:3 passage (a density comparable to general maintenance passage on matrigel-coated tissue culture plastic) in basal media ( $\alpha$-MEM (Sigma) supplemented with 10\% FCS (Invitrogen) and penicillin/streptomycin (Lonza)). Media was changed twice weekly. Cells were harvested, lysed and RNA/DNA isolated using AllPrep RNA/DNA Kit (Qiagen).

Embryoid Bodies: Embryoid bodies (EBs) were formed by manual scraping of day 3 hESCs cultured on MEFs. Dissociated cells were transferred to non-adherent stereological plates in Knockout DMEM supplemented with 10\% FCS, glutamine (1 mM), penicillin/streptomycin $(100 \mu \mathrm{g} / \mathrm{mL})$, MEM non-essential amino acids $(0.1 \mathrm{mM})$ and $\beta$-mercaptoethanol $(3.5 \mu \mathrm{M})$. Plates were placed on a swirling platform at $37{ }^{\circ} \mathrm{C}$ for 4 days. EBs were collected and resuspended in basal media prior to seeding nanotopography substrates.

Immunofluorescence: Live dead staining was conducted using Cell Tracker Green CMFDA (5-chloromethylfluorescein diacetate)
(C7025, Invitrogen) and ethidium homodimer-1 (E1169, Invitrogen). For immunofluorescent staining, cells were fixed directly on matrigel-coated tissue culture plastic, planar or NSq50 substrates using $4 \%$ paraformaldehyde. Following 10 min incubation, cells were washed with PBS and incubated for 10 min with permeabilisation buffer ( $0.1 \%$ Triton $X$ in PBS). Cells were blocked with $1 \%$ BSA (Sigma-Aldrich) in PBS for 5 min. Primary antibodies BMP4 (Abcam39973), CD44 (Protein Tech 15675-1-AP), type I collagen (LF 68, Dr. Larry Fisher, NIH, Bethesda, MD), E-cadherin (Abcam ab15148), N-cadherin (Abcam ab12221), nestin (Santa Cruz 20978) ( $\alpha$-nestin antibody was tested on mouse brain sections), OCT4 (Abcam 19857), SLUG/SNAIL (Abcam ab85931), SOX2 (Millipore AB5603), SSEA4 (Abcam 16287), STRO1 (hybridoma supernatant, Dr. J. Beresford, University of Bath), TRA-1-60 (Abcam 16288) or appropriate isotype control antibodies were diluted in $1 \%$ BSA blocking buffer and applied to cells overnight at $4{ }^{\circ} \mathrm{C}$. Three washes with PBS Tween $(0.5 \%)$ for 5 min each were conducted prior to applying secondary antibody (Alexa fluor 488) diluted in BSA (1\%) blocking buffer. Nuclei were counter stained with 1:200 DAPI (4',6-diamidino-2-phenylindole) (D3571, Invitrogen) in PBS for 5 min followed by three washes with PBS Tween for 5 minutes each. All images were captured on a Carl Zeiss Axiovert 200 microscope with Axiovision software (version 4.5) via an AxioCam HR digital camera for phase images or via an AxioCam MRc with appropriate filters for fluorescence microscopy. Fluorescence intensity was quantified using Image J software $(\mathrm{NIH})$ and is represented graphically as mean fluorescence intensity $(n=3)$ with standard deviation.

Quantitative PCR: Isolated RNA was DNase treated and reverse transcribed using Super-Script First-strand synthesis system for PCR (Invitrogen). Assessment of marker expression was conducted by quantitative PCR (qPCR) using Power Sybr Green PCR Master Mix (Applied Biosystems). Forward and reverse primers used for $\mathrm{qPCR}$ are listed in Table S1. For all experiments, data are represented as relative fold change in expression following normalisation to $\beta$-Actin with standard error of the mean (SEM).

PCR Arrays: Isolated RNA was converted to cDNA using RT2 first strand kit (330401, SA Biosciences) according to the manufacturer's instructions. Subsequently, cDNA was mixed with RT2 qPCR master mix and aliquots transferred to 96 well mesenchymal stem cell (MSC) PCR array plates (PAHS-082, SA Biosciences). Three independent experiments were conducted and data were analysed using the online RT2 Profiler PCR Array Data Analysis (SA Biosciences) tool by normalising to GAPDH and $\beta$-Actin housekeeping genes and subsequently represented as fold regulation compared to self-renewing hESC samples (hESCs maintained on matrigel in CM) or day 14 planar control samples as indicated in figure legends. Cluster analysis was performed on mean $\mathrm{Ct}$ values normalised to $\beta$-Actin using MatLab (Version 7).

Global Methylation Analysis: Genomic DNA was used to investigate global methylation using MethylFlash Methylated DNA Quantification Kit (Epigentek) according to the manufacturer's instructions. DNA from three independent experiments is represented as a mean percentage of relative global methylation levels with SEM.

Promoter Methylation Analysis: Genomic DNA was subjected to bisulfite conversion using EZ DNA Methylation-Gold Kit (D5005, Zymo). Promoter regions were amplified using specifically designed primers (Table S2) and Platinum PCR Supermix (11306016, 
Invitrogen). Methylation was quantified by pyrosequencing and is expressed as the percentage of methylated cytosine residues at a particular CpG site of total methylated (cytosine residues not converted during bisulfite modification) and unmethylated (cytosine residues converted to uracil and thymine during bisulfite modification and PCR respectively) residues at this position. Mean percentage methylation is shown with SEM.

Statistics: GraphPad Prism 5 statistical software was used to perform statistical analysis. Kruskal-Wallis test and Dunn's multiple comparisons post test were used to determine statistical significance for qPCR data. Analysis of variance with Tukey post test was used to determine statistical significance for all other data. $P$ values for statistical significance are indicated where $*$ indicates $\mathrm{p}<0.05, * *$ indicates $\mathrm{p}<0.01$ and $* * *$ indicates $\mathrm{p}<0.001$. Details. 12 point, double-spaced.

\section{Supporting Information}

Supporting Information is available from the Wiley Online Library or from the author.

\section{Acknowledgements}

This work was funded by a BBSRC grant G006970/1. The authors gratefully acknowledge Mary Robertson, Bill Monaghan and Kate Parry for technical support and Franchesca Houghton for critical reading of the manuscript.

[1] J. Thomson, J. Itskovitz-Eldor, S. Shapiro, M. Waknitz, J. Swiergiel, V. Marshall, J. Jones, Science 1998, 282, 1145.

[2] J. I. Dawson, R. O. Oreffo, Arch. Biochem. Biophys. 2008, 473, 124.

[3] A. Yamashita, S. Nishikawa, D. E. Rancourt, PLoS One 2010, 5, e9663.

[4] a) N. I. zur Nieden, G. Kempka, D. E. Rancourt, H. J. Ahr, BMC Dev. Biol. 2005, 5, 1; b) V. Sottile, A. Thomson, J. McWhir, Cloning Stem Cells 2003, 5, 149; c) T. Cao, B. C. Heng, C. P. Ye, H. Liu, W. S. Toh, P. Robson, P. Li, Y. H. Hong, L. W. Stanton, Tissue Cell 2005, 37, 325.

[5] J. M. Karp, L. S. Ferreira, A. Khademhosseini, A. H. Kwon, J. Yeh, R. S. Langer, Stem Cells 2006, 24, 835.

[6] M. Dalby, N. Gadegaard, R. Tare, A. Andar, M. Riehle, P. Herzyk, C. Wilkinson, R. Oreffo, Nat. Mater. 2007, 6, 997.

[7] R. J. McMurray, N. Gadegaard, P. M. Tsimbouri, K. V. Burgess, L. E. McNamara, R. Tare, K. Murawski, E. Kingham, R. O. Oreffo, M. J. Dalby, Nat. Mater. 2011, 10, 637.

[8] P. M. Tsimbouri, R. J. McMurray, K. V. Burgess, E. V. Alakpa, P. M. Reynolds, K. Murawski, E. Kingham, R. O. Oreffo, N. Gadegaard, M. J. Dalby, ACS Nano 2012, 6, 10239.

[9] O. F. Zouani, C. Chanseau, B. Brouillaud, R. Bareille, F. Deliane, M. P. Foulc, A. Mehdi, M. C. Durrieu, J. Cell Sci. 2012, 125, 1217.

[10] S. Oh, K. S. Brammer, Y. S. Li, D. Teng, A. J. Engler, S. Chien, S. Jin, Proc. Natl. Acad. Sci. USA 2009, 106, 2130.

[11] a) E. Garreta, E. Genové, S. Borrós, C. Semino, Tissue Eng. 2006, 12, 2215; b) L. Smith, X. Liu, J. Hu, P. Wang, P. Ma, Tissue Eng. Part A 2009, 15, 1855; c) L. Smith, X. Liu, J. Hu, P. Ma, Biomaterials
2009, 30, 2516; d) L. A. Smith, X. Liu, J. Hu, P. X. Ma, Biomaterials 2010, 31, 5526 .

[12] a) I. Muñoz-Sanjuán, A. Brivanlou, Nat. Rev. Neurosci. 2002, 3, 271 ; b) I. Bouhon, H. Kato, S. Chandran, N. Allen, Brain Res. Bull. 2005, 68, 62 .

[13] a) H. Kawasaki, K. Mizuseki, S. Nishikawa, S. Kaneko, Y. Kuwana, S. Nakanishi, S. I. Nishikawa, Y. Sasai, Neuron 2000, 28, 31; b) K. Watanabe, D. Kamiya, A. Nishiyama, T. Katayama, S. Nozaki, H. Kawasaki, Y. Watanabe, K. Mizuseki, Y. Sasai, Nat. Neurosci. 2005, 8, 288; c) S. R. Smukler, S. B. Runciman, S. Xu, D. van der Kooy, J. Cell Biol. 2006, 172, 79.

[14] a) W. Kafienah, S. Mistry, C. Williams, A. P. Hollander, Stem Cells 2006, 24, 1113; b) S. J. Liu, Z. W. Cai, Y. J. Liu, M. Y. Dong, L. Q. Sun, G. F. Hu, Y. Y. Wei, W. D. Lao, World J. Gastroenterol. 2004, 10, 1246; c) R. Y. Tsai, R. D. McKay, Genes Dev. 2002, 16, 2991.

[15] K. Stewart, P. Monk, S. Walsh, C. M. Jefferiss, J. Letchford, J. N. Beresford, Cell Tissue Res. 2003, 313, 281.

[16] S. Gronthos, S. E. Graves, S. Ohta, P. J. Simmons, Blood 1994, 84, 4164.

[17] a) R. Sackstein, J. S. Merzaban, D. W. Cain, N. M. Dagia, J. A. Spencer, C. P. Lin, R. Wohlgemuth, Nat. Med. 2008, 14, 181; b) J. B. Mitchell, K. McIntosh, S. Zvonic, S. Garrett, Z. E. Floyd, A. Kloster, Y. Di Halvorsen, R. W. Storms, B. Goh, G. Kilroy, X. Wu, J. M. Gimble, Stem Cells 2006, 24, 376.

[18] P. Ducy, R. Zhang, V. Geoffroy, A. L. Ridall, G. Karsenty, Cell 1997, $89,747$.

[19] J. D. Termine, H. K. Kleinman, S. W. Whitson, K. M. Conn, M. L. McGarvey, G. R. Martin, Cell 1981, 26, 99.

[20] a) M. E. Greene, J. Pitts, M. A. McCarville, X. S. Wang, J. A. Newport, C. Edelstein, F. Lee, S. Ghosh, S. Chu, Prostaglandins Other Lipid Mediat. 2000, 62, 45; b) P. Tontonoz, E. Hu, R. A. Graves, A. I. Budavari, B. M. Spiegelman, Genes Dev. 1994, 8, 1224.

[21] Y. S. Chen, R. A. Pelekanos, R. L. Ellis, R. Horne, E. J. Wolvetang, N. M. Fisk, Stem Cells Transl. Med. 2012, 1, 83.

[22] J. M. Lee, S. Dedhar, R. Kalluri, E. W. Thompson, J. Cell Biol. 2006, 172, 973.

[23] D. Evseenko, Y. Zhu, K. Schenke-Layland, J. Kuo, B. Latour, S. Ge, J. Scholes, G. Dravid, X. Li, W. R. MacLellan, G. M. Crooks, Proc. Natl. Acad. Sci. USA 2010, 107, 13742.

[24] V. Bolós, H. Peinado, M. A. Pérez-Moreno, M. F. Fraga, M. Esteller, A. Cano, J. Cell Sci. 2003, 116, 499.

[25] K. M. Hajra, D. Y. Chen, E. R. Fearon, Cancer Res. 2002, 62, 1613.

[26] E. Batlle, E. Sancho, C. Francí, D. Domínguez, M. Monfar, J. Baulida, A. García De Herreros, Nat. Cell Biol. 2000, 2, 84.

[27] A. Cano, M. A. Pérez-Moreno, I. Rodrigo, A. Locascio, M. J. Blanco, M. G. del Barrio, F. Portillo, M. A. Nieto, Nat. Cell Biol. 2000, 2, 76.

[28] M. A. Nieto, M. G. Sargent, D. G. Wilkinson, J. Cooke, Science $1994,264,835$.

[29] A. M. Eastham, H. Spencer, F. Soncin, S. Ritson, C. L. Merry, P. L. Stern, C. M. Ward, Cancer Res. 2007, 67, 11254.

[30] D. Baksh, G. M. Boland, R. S. Tuan, J. Cell Biochem. 2007, 101, 1109.

[31] A. Yamashita, T. Takada, K. Nemoto, G. Yamamoto, R. Torii, FEBS Lett. 2006, 580, 4121.

[32] S. Yeo, S. Jeong, J. Kim, J. S. Han, Y. M. Han, Y. K. Kang, Biochem. Biophys. Res. Commun. 2007, 359, 536.

[33] K. Hosoda, S. Kanzaki, H. Eguchi, M. Kiyoki, T. Yamaji, Y. Koshihara, M. Shiraki, Y. Seino, J. Bone Miner. Res. 1993, 8, 553.

[34] M. C. de Andres, E. Kingham, K. Imagawa, A. Gonzalez, H. I. Roach, D. I. Wilson, R. O. C. Oreffo, PLoS One 2013, DOI: 10.1371/journal.pone.0054957.

[35] A. Engler, S. Sen, H. Sweeney, D. Discher, Cell 2006, 126, 677.

[36] R. McBeath, D. Pirone, C. Nelson, K. Bhadriraju, C. Chen, Dev. Cell 2004, 6, 483.

[37] K. A. Kilian, B. Bugarija, B. T. Lahn, M. Mrksich, Proc. Natl. Acad. Sci. USA 2010, 107, 4872. 
[38] D. S. Benoit, M. P. Schwartz, A. R. Durney, K. S. Anseth, Nat. Mater. 2008, 7, 816; J. M. Curran, R. Chen, J. A. Hunt, Biomaterials 2006, 27, 4783.

[39] J. T. Taiani, R. J. Krawetz, N. I. Zur Nieden, Y. Elizabeth Wu, M. S. Kallos, J. R. Matyas, D. E. Rancourt, Stem Cells Dev. 2010, $19,989$.

[40] E. J. Gang, D. Bosnakovski, C. A. Figueiredo, J. W. Visser, R. C. Perlingeiro, Blood 2007, 109, 1743.

[41] P. V. Guillot, C. Gotherstrom, J. Chan, H. Kurata, N. M. Fisk, Stem Cells 2007, 25, 646.

[42] W. Wojakowski, M. Tendera, M. Kucia, E. Zuba-Surma, E. Paczkowska, J. Ciosek, M. Hałasa, M. Król, M. Kazmierski, P. Buszman, A. Ochała, J. Ratajczak, B. Machaliński, M. Z. Ratajczak, J. Am. Coll. Cardiol. 2009, 53, 1.

[43] S. Pringle, C. De Bari, F. Dell'Accio, S. Przyborski, M. J. Cooke, S. L. Minger, A. E. Grigoriadis, Cell Prolif. 2011, 44, 120.

[44] E. Kärner, C. Unger, A. J. Sloan, L. Ahrlund-Richter, R. V. Sugars, M. Wendel, Stem Cells Dev. 2007, 16, 39.

[45] F. Chowdhury, S. Na, D. Li, Y. C. Poh, T. S. Tanaka, F. Wang, N. Wang, Nat. Mater. 2010, 9, 82.

[46] M. Kanai-Azuma, Y. Kanai, J. M. Gad, Y. Tajima, C. Taya, M. Kurohmaru, Y. Sanai, H. Yonekawa, K. Yazaki, P. P. Tam, Y. Hayashi, Development 2002, 129, 2367.
[47] K. A. D’Amour, A. D. Agulnick, S. Eliazer, O. G. Kelly, E. Kroon, E. E. Baetge, Nat. Biotechnol. 2005, 23, 1534.

[48] W. J. Anderson, Q. Zhou, V. Alcalde, O. F. Kaneko, L. J. Blank, R. I. Sherwood, J. S. Guseh, J. Rajagopal, D. A. Melton, Dev. Dyn. 2008, 237, 504.

[49] B. G. Herrmann, A. Kispert, Trends Genet. 1994, 10, 280.

[50] E. Gentleman, R. J. Swain, N. D. Evans, S. Boonrungsiman, G. Jell, M. D. Ball, T. A. Shean, M. L. Oyen, A. Porter, M. M. Stevens, Nat. Mater. 2009, 8, 763.

[51] Z. Qin, L. Kreplak, M. J. Buehler, PLoS One 2009, 4, e7294.

[52] Y. Nakamura, R. Hashimoto, M. Amano, K. Nagata, N. Matsumoto, H. Goto, E. Fukusho, H. Mori, Y. Kashiwagi, T. Kudo, M. Inagaki, M. Takeda, Genes Cells 2000, 5, 823.

[53] W. Chen, L. G. Villa-Diaz, Y. Sun, S. Weng, J. K. Kim, R. H. Lam, L. Han, R. Fan, P. H. Krebsbach, J. Fu, ACS Nano 2012, 6, 4094.

[54] Y. Sun, L. G. Villa-Diaz, R. H. Lam, W. Chen, P. H. Krebsbach, J. Fu, PLoS One 2012, 7, e37178.
Received: September 24, 2012 Revised: November 1, 2012 Published online: 